Acta Bot. Croat. $72(1), 49-69,2013$

\title{
Karyological and morphological variations within the genus Dysphania (Chenopodiaceae) in Bulgaria
}

\author{
Neli H. Grozeva ${ }^{1}$, Yanka G. Cvetanova ${ }^{2}$ \\ ${ }^{1}$ Department of Biology and Aquaculture, Agriculture Faculty, Trakia University, \\ Stara Zagora, Bulgaria \\ ${ }^{2}$ Department of Informatics and Mathematics, Faculty of Economics, Trakia University, \\ Stara Zagora, Bulgaria
}

\begin{abstract}
The karyological and morphological variability of species from the genus Dysphania were studied. The results demonstrated that genus Dysphania is represented in Bulgaria by five species: Dysphania ambrosioides, D. multifida, D. botrys, D. schraderi$a n a$ and $D$. pumilio. The first two species are tetraploids with chromosome number $2 n=32$ for $D$. ambrosioides and $2 n=36$ for $D$. multifida. The remaining three species are diploids with $2 n=18$. The results from statistical analysis demonstrated that the main source of phenotype variation in the species is the interpopulation variation. The specific characters which allowed their recognition are the morphological characteristics of the perianth lobes, the upper leaves and the seeds. The distinction between D. multifida, D. ambrosioides and $D$. schraderiana is based on differences in the quantitative traits, while in $D$. botrys and $D$. pumilio qualitative traits are also important. The basic evolutionary mechanisms are polyploidy and diploidy. A tendency towards reduction in the size of generative organs and the number of perianth lobes was found.
\end{abstract}

Key words: Dysphania, distribution, ecology, karyology, morphology, Bulgaria

\section{Introduction}

Traditionally, the genus Dysphania comprises 6-10 species, endemic for Australia (Mosyakin 1993, Clements and Mosyakin 2003, Zhu et al. 2003). Over the past decades, the systemic definition and composition of the genus has frequently been a subject of discussion (MosYAKIN 1993; MosYAKIN and CLEMENTS 1996, 2002, 2008). Currently the genus Dysphania comprises about 40 species that earlier constituted the subgenus Ambrosia of the genus Chenopodium or were referred to the genera: Neobotritium Moldenke, Roubieva Moquin-Tandon, Teloxys Moquin-Tandon. The representatives of the genus are mainly ruderal and weed plants, more common in the tropics, subtropics and warm-temperature

* Corresponding author, e-mail: grozeva@uni-sz.bg

Copyright $^{\circledR} 2013$ by Acta Botanica Croatica, the Faculty of Science, University of Zagreb. All rights reserved. 
zone. Most of them entered Europe through the import of wool and agricultural products from Australia, South America, Eastern Asia, and Africa. A number of researchers (AELLEN 1960, Uotila and SuOMinen 1976, Dostalek 1985, KüHn 1993, Uotila 2001) pointed out their great morphological variability, especially prominent in the leaf lamina. The similar, rarely exceeding $1 \mathrm{~mm}$, dimensions of the generative organs very often make their distinction difficult.

For the Bulgarian flora so far five species have been reported - Chenopodium ambrosioides L. (= Dysphania ambrosioides (L.) Mosyakin et Clements), C. botrys L. (= Dysphania botrys (L.) Mosyakin et Clements), C. multifidum L. (= Dysphania multifida (L.) Mosyakin et Clements), C. pumilio R. Br. (= Dysphania pumilio (R. Br.) Mosyakin et Clements) and C. schraderianum Schult. (= Dysphania schraderiana (Schult.) Mosyakin et Clements) (Markova 1966, Assyov and Petrova 2006, Grozeva 2007).

Dysphania ambrosioides forms populations on open sandy terrain, in close proximity to water basins from sea level to $250 \mathrm{~m}$ above sea level. It is usually a dominant species in anthropophyte or ruderal communities.

D. multifida forms populations on open lowland areas, more rarely on those facing west or south at altitudes from sea level to $700 \mathrm{~m}$ above sea level. It dominates or is an accompanying species in anthropophyte or ruderal communities dominated by cereal species.

$D$. botrys is widely spread on open, sunlit areas and as a weed in cultivated crops at altitudes from sea level to $900 \mathrm{~m}$ above sea level. Most often, it dominates or is an accompanying species in ruderal communities.

D. schraderiana was established for the Bulgarian flora by Pertti Uotila in 1993 while revising the materials from the genus Chenopodium in the herbarium of the Biological Faculty at Sofia University (SO). The species has very limited distribution. So far, only two populations have been known located in Vitosha and in Sredna Gora Mountain. Its ecological requirements are quite similar to these of $D$. botrys and specimens from both species were recorded in the population under study.

D. pumilio occurs in single localities in the Eastern Balkan Range, Thracian Lowland, Northeast Bulgaria, Black Sea Coast and Tundzha Hilly Country as an accompanying species in ruderal communities (Grozeva 2007, Vladimirov and Petrova 2010, Vladimirov 2011). The species forms populations in open sunlit places at an altitude from 168 to $850 \mathrm{~m}$.

The objective of this study was to investigate the patterns and levels of morphological variation, chromosome numbers and karyotype morphology as well as ecological conditions of the natural local Bulgarian populations of genus Dysphania, and to attempt to trace the relationships between the species and some evolutionary trends in the genus.

\section{Materials and methods}

Morphological and karyological analyses were carried out on 27 natural Bulgarian populations of the genus Dysphania, referred to five species: Dysphania ambrosioides, D. botrys, $D$. multifida, D. pumilio, and $D$. schraderiana. Thirty plants from each population were used in the overall research.

Chromosome numbers and karyotypes have been reported on lasting preparations of metaphase root apex plates of seeds germinated in laboratory conditions collected in the nat- 
ural habitats of the species. The root tips were treated and squashed according to the accepted methods (Grozeva 2007). The chromosomal type was determined after the centromere index I $=\mathrm{s} / \mathrm{s}+1$, according to the classification proposed by GRIF and AGAPOVA (1986). Three metaphase plates have been measured from each population. The voucher specimens are kept in the herbarium of the Bulgarian Academy of Sciences (SOM).

Twenty-four quantitative characters were included in the morphological analysis: 1 . Height of plant; 2. Length of basal leaf; 3. Width of basal leaf; 4. Length/width ratio; 5. Length of basal leaf petiole; 6 . Length of upper leaf; 7 . Width of upper leaf; 8 . Length/width ratio; 9 . Length of upper leaf petiole; 10 . Length of inflorescence; 11 . Length of flower petiole; 12. Diameter of flower; 13. Length of perianth lobes in bisexual flower; 14. Width of perianth lobes in bisexual flower; 15 . Length of perianth lobes in female flower; 16 . Width of perianth lobes in female flower; 17. Length of seed; 18 . Width of seed; 19. Length/width ratio; 20. Thickness of seed; 21. Length of fruit; 22. Width of fruit; 23. Length/width ratio; 24. Thickness of fruit. The following qualitative features were employed too: colour of stem; type of inflorescence; degree of perianth concrescence; presence of keeled perianth lobes; colour of perianth, seed and pericarp.

The mean value and coefficient of variation were calculated for each character of every population. They were used in the comparative analyses on different levels.

The relative contributions of intra- and interpopulation variation to the overall variation of each characteristic of the studied species were evaluated by one-way ANOVA.

Unweighted pair-group average (UPGA) hierarchical cluster analysis (HCA) was applied to the matrix with the Euclidean distances between the populations of the genus Dysphania in order to study the morphological pair-wise similarities and the underlying hierarchical classification structure.

The discriminant function analysis was conducted on morphological data to determine the most parsimonious way to distinguish between species. The stepwise discriminant analysis (SDA) procedure was used as it allows recognition of the most effectively discriminating variables among a large set of morphological characters.

Statistica 6.0 (StatSoft Inc. 2001) was used for the statistical analysis of morphological data.

\section{Results}

\section{Karyology}

As a result of karyological study, 3 chromosome numbers: $2 n=18,32,36$ have been found in the genus Dysphania (Tab. 1). Two types of chromosomes: metacentric and submetacentric have been established in the karyotypes.

The number $2 n=18$ has been counted in three species - Dysphania botrys, D. pumilio and $D$. schraderiana. The last species was studied karyologically in Bulgaria for the first time and for the studied population karyotype $2 n=14 \mathrm{~m}+4 \mathrm{sm}=18$ chromosomes has been established (Plate 1A). The diploid chromosome number found for D. schraderiana confirms the data known from literature sources (KJELLMARK 1934, SCHWARZOVA 1978a). For all three populations of $D$. pumilio the karyotype of $2 n=8 \mathrm{~m}+10 \mathrm{sm}=18$ chromosomes has been recorded (Plate 1B). The chromosome number $2 n=18$ found for the Bulgarian popu- 
Tab. 1. Description of studied populations of genus Dysphania. * - data published by GROZEVA and StOEVA (2006). ** - data published by GROZEVA (2007)

\begin{tabular}{ccc}
\hline $\begin{array}{c}\text { Section, species } \\
\text { and population } \\
\text { number }\end{array}$ & $2 n \quad$ Locality \\
\hline
\end{tabular}

Sect. Adenois (Moq.) Mosyakin et Clements.

Dysphania ambrosioides (L.) Mosyakin et Clemants

№201 32** Danubian plain, island Milka near Belene town, at $27 \mathrm{~m}, 43^{\circ} 40^{\prime} \mathrm{N}$, $25^{\circ} 10^{\prime} \mathrm{E}$, in sandy hair

№32 32** Thracian Lowland, Plovdiv town, near the river Maritza, at $164 \mathrm{~m}$, $42^{\circ} 09^{\prime} \mathrm{N}, 24^{\circ} 45^{\prime} \mathrm{E}$, in ruderal places

№104 32** Thracian Lowland, the locality Ostrova near Plovdiv town, at $164 \mathrm{~m}$, $42^{\circ} 09^{\prime} \mathrm{N}, 24^{\circ} 45^{\prime} \mathrm{E}$, in ruderal places

Dysphania multifida (L.) Mosyakin et Clemants

№39 36 Danubian plain, Island Goljama Barzina near Belene town, at $20 \mathrm{~m}$, $43^{\circ} 40^{\prime} \mathrm{N}, 25^{\circ} 10^{\prime} \mathrm{E}$, in outskirts of the island and on sandy hair

№40 36 Danubian plain, Vidin town, at $345 \mathrm{~m}, 43^{\circ} 59^{\prime} \mathrm{N}, 22^{\circ} 52^{\prime} \mathrm{E}$, in ruderal places near the bus station with Trifolium album and other legumes.

№105 36 Central Balkan Range, Kalofer town, at 666 m, 42 37’ N,24 59’ E, grasslands with Lolium perenne L. of $50 \mathrm{~m}$ from post

№106 36** Western Sredna Gora Mt., Ikhtiman town, at $658 \mathrm{~m}, 42^{\circ} 09^{\prime} \mathrm{N}$, $24^{\circ} 45^{\prime} \mathrm{E}$, in ruderal places in the western parts of the city together with cereal species

№107 36** Eastern Rhodope Mts, Ivaylovgrad town, at $104 \mathrm{~m}, 41^{\circ} 32^{\prime} \mathrm{N}, 26^{\circ} 08^{\prime} \mathrm{E}$, in ruderal places together with Bromus tectorum L. and Setaria viridis

№108 36 Thracian Lowland, Purvomay town, at $134 \mathrm{~m}, 42^{\circ} 06^{\prime} \mathrm{N}, 2^{\circ} 13^{\prime} \mathrm{E}$, grasslands in kvartal Debar.

№109 36** Thracian Lowland, Asenovgrad town, at $104 \mathrm{~m}, 42^{\circ} 01^{\prime} \mathrm{N}, 2^{\circ}{ }^{\circ} 52^{\prime} \mathrm{E}$, in ruderal places together with Setaria viridis

Sect. Botryoides (C. A. Mey) Mosyakin et Clemants

Dysphania botrys (L.) Mosyakin et Clemants

№29 18 Northern Black Sea Coast, around Varnensko ezero lake, at $79 \mathrm{~m}$, $43^{\circ} 11^{\prime} \mathrm{N}, 27^{\circ} 50^{\prime} \mathrm{E}$, periodically flooded coastal alluvial sands deposits

№97 18 Southern Black Sea Coast, Pomorie town, at $0 \mathrm{~m}, 42^{\circ} 35^{\prime} \mathrm{N}, 2^{\circ} 37^{\prime} \mathrm{E}$, in ruderal places near Monastery of Saint George

№100 18** North-Eastern Bulgaria, Razgrad town, at $200 \mathrm{~m}, 43^{\circ} 46^{\prime} \mathrm{N}, 26^{\circ} 31^{\prime} \mathrm{E}$, between the sidewalk and fences together with Polygonum aviculare

№35 18** Danubian plain, Belene town, at $35 \mathrm{~m}, 43^{\circ} 39^{\prime} \mathrm{N}, 25^{\circ} 07^{\prime} \mathrm{E}$, in ruderal places near the fishing pier

№102 18 Easthern Balkan Range, Natural park »Sinite kamani«, at 290 m, $42^{\circ} 42^{\prime} \mathrm{N}, 26^{\circ} 21^{\prime} \mathrm{E}$, in ruderal places near road from hotel Ablanovo to the lake Asenovets

№99 18** Central Balkan Range, Trojan town, at $400 \mathrm{~m}, 43^{\circ} 53^{\prime} \mathrm{N}, 24^{\circ} 43^{\prime} \mathrm{E}$, in ruderal places from $50 \mathrm{~m}$ to bus station 
Tab. 1. - continued

\begin{tabular}{|c|c|c|}
\hline $\begin{array}{l}\text { Section, species } \\
\text { and population } \\
\text { number }\end{array}$ & $2 n$ & Locality \\
\hline №98 & 18 & $\begin{array}{l}\text { Sofia region, Malo Buchino village, at } 748 \mathrm{~m}, 42^{\circ} 41^{\prime} \mathrm{N}, 23^{\circ} 10^{\prime} \mathrm{E} \text {, } \\
\text { along the sidewalk between the plates together with Polygonum } \\
\text { aviculare and Cynodon dactylon (L.) Pers. }\end{array}$ \\
\hline №101 & 18 & $\begin{array}{l}\text { Struma valley, Blagoevgrad town, at } 360 \mathrm{~m}, 41^{\circ} 45^{\prime} \mathrm{N}, 23^{\circ} 25^{\prime} \mathrm{E} \text {, weed } \\
\text { in the cultural community }\end{array}$ \\
\hline №53 & 18 & $\begin{array}{l}\text { Easthern Sredna Gora Mt., Gorno Novo selo village, at } 597 \mathrm{~m} \text {, } \\
42^{\circ} 27^{\prime} \mathrm{N}, 25^{\circ} 14^{\prime} \mathrm{E} \text {, in ruderal places near the road for a hut } \\
\text { Kavakliika together with Cynodon dactylon. }\end{array}$ \\
\hline №30 & 18 & $\begin{array}{l}\text { Thracian Lowland, Plovdiv town, near the river Maritza, at } 164 \mathrm{~m} \text {, } \\
42^{\circ} 09^{\prime} \mathrm{N}, 24^{\circ} 45^{\prime} \mathrm{E} \text {, in ruderal places }\end{array}$ \\
\hline №34 & 18 & $\begin{array}{l}\text { Thracian Lowland, Malka Vereja village, at } 235 \mathrm{~m}, 42^{\circ} 24^{\prime} \mathrm{N}, 25^{\circ} 33^{\prime} \mathrm{E} \text {, } \\
\text { in ruderal places at the eastern edge of the village together with } \\
\text { Atriplex tatarica L. and Setaria viridis (L.) Beauv }\end{array}$ \\
\hline №45 & 18 & $\begin{array}{l}\text { Thracian Lowland, Tselina village, at } 193 \mathrm{~m}, 42^{\circ} 07^{\prime} \mathrm{N} 25^{\circ} 27^{\prime} \mathrm{E} \text {, weed } \\
\text { in the cultural community }\end{array}$ \\
\hline \multicolumn{3}{|c|}{ Dysphania schraderiana (Schult.) Mosyakin et Clemants } \\
\hline №103 & 18 & $\begin{array}{l}\text { Easthern Sredna Gora Mt., near Kavakliika hut, } \\
\text { at } 650 \mathrm{~m}, 42^{\circ} 27^{\prime} \mathrm{N}, 25^{\circ} 12^{\prime} \mathrm{E} \text {, in ruderal places around the hut of } \\
\text { forestry together with Dysphania botrys }\end{array}$ \\
\hline \multicolumn{3}{|c|}{ Sect. Orthospora (R. Br.) Mosyakin et Clemants } \\
\hline \multicolumn{3}{|c|}{ Dysphania pumilio (R. Br.) Mosyakin et Clemants } \\
\hline №214 & $18 *$ & $\begin{array}{l}\text { Eastern Balkan Range, Sinite kamani Natural Park, at } 850 \mathrm{~m}, 42^{\circ} 42^{\prime} \mathrm{N} \text {, } \\
25^{\circ} 20^{\prime} \mathrm{E} \text {, in ruderal places by roadsides together with Polygonum } \\
\text { aviculare L. }\end{array}$ \\
\hline №-217 & 18 & $\begin{array}{l}\text { Thracian Lowland, Stara Zagora town, at } 195 \mathrm{~m}, 42^{\circ} 25^{\prime} \mathrm{N}, 25^{\circ} 38^{\prime} \mathrm{E} \text {, } \\
\text { in ruderal places together with Dysphania botrys }\end{array}$ \\
\hline №221 & 18 & $\begin{array}{l}\text { Thracian Lowland, Chirpan town, at } 168 \mathrm{~m}, 42^{\circ} 25^{\prime} \mathrm{N}, 25^{\circ} 38^{\prime} \mathrm{E} \text {, in } \\
\text { ruderal places together with cereal plants }\end{array}$ \\
\hline
\end{tabular}

lations of $D$. pumilio corresponds to the data of JAVOURKOVA-JAROMIROVA (1992) and MesíčEK (1992) from the Czech Republic, SchwARZovÁ (1978b) from Slovakia and RAHIMENIJAD (2004) from Iran. With the third diploid species D. botrys differences have been recorded in the karyotype of the various populations. In population No. 29 from the Varna lake and population No. 30 from Plovdiv the karyotype consists of $2 n=4 \mathrm{~m}+14 \mathrm{sm}=$ 18 chromosomes (Plate 1C). In population No. 99 from Troyan and population No. 45 from Tselina satellites can be seen on one pair of submetacentric chromosomes (Plate 1D). In the remaining eight populations (Tab. 1), a karyotype of 18 submetacentric chromosomes has been established. In four of these populations (No. 97 from Pomorie; No. 102 from »Sinite kamani« Natural Park; No. 53 from Gorno Novo selo; No. 100 from Razgrad) one of the chromosome pairs has satellites (Plate 2A), while in the other four (No. 35 from Belene; No. 
Grozeva N. H., Cvetanova Y. G.
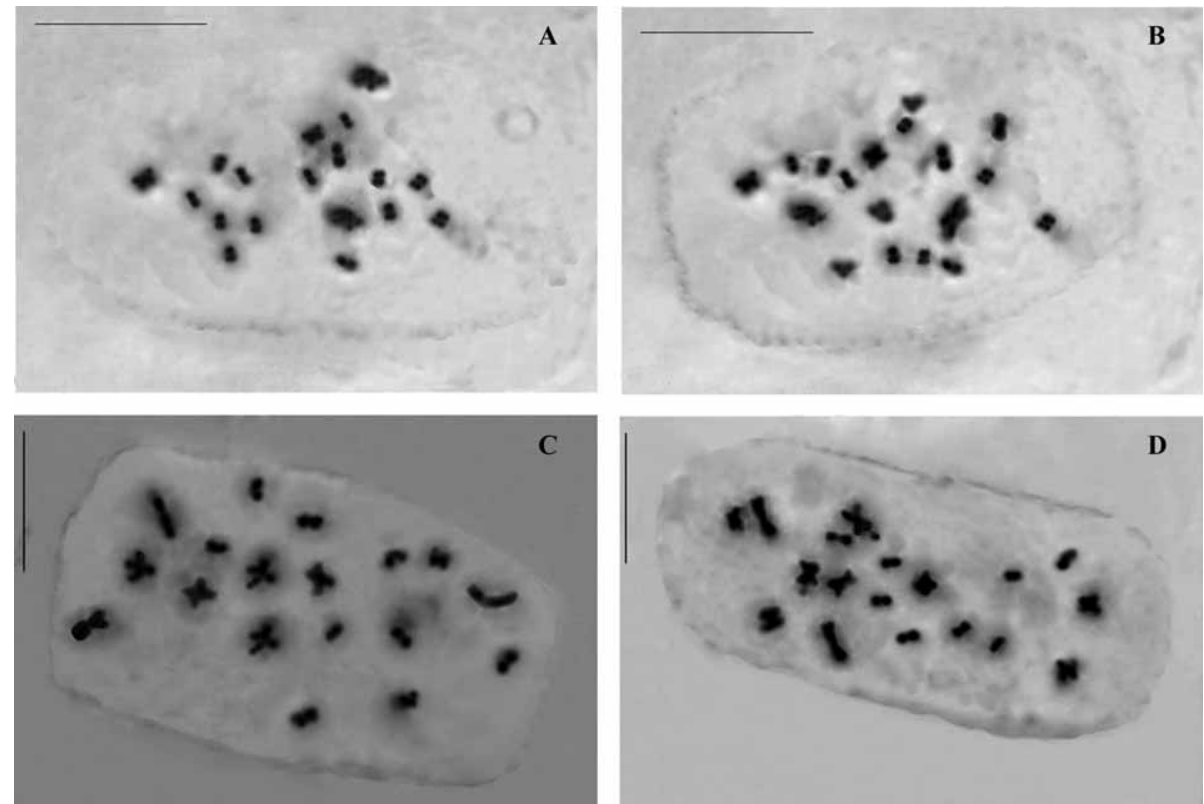

Plate 1. Microphotographs of the metaphase plate of Dysphania species: A - D. schraderiana (population № 103) $-2 n=18 ; \mathbf{B}-D$. pumilio (№ 221) $-2 n=18 ; \mathbf{C}-D$. botrys (№ 29) $-2 n=18$; D - D. botrys (№ 45) $-2 n=18$. - Scale bars $=10 \mu \mathrm{m}$.
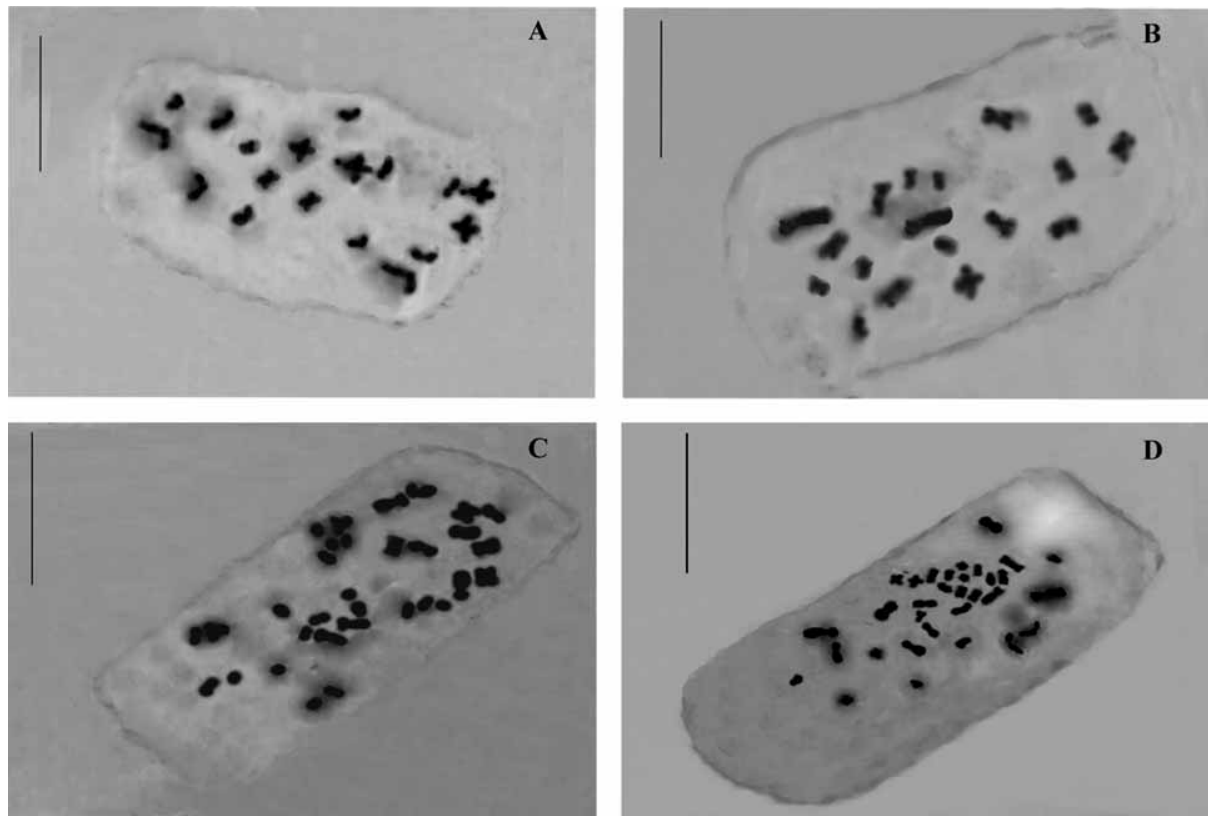

Plate 2. Microphotographs of the metaphase plate of Dysphania species: A-D. botrys (population № $53)-2 n=18 ; \mathbf{B}-D$. botrys (№ 98) $-2 n=18 ; \mathbf{C}-D$. ambrosioides (№ 102) $-2 n=32 ; \mathbf{D}-D$. ambrosioides (№ 201) $-2 n=32$. - Scale bars $=10 \mu \mathrm{m}$. 
98 from Malo Buchino; No. 34 from Malka Vereya; No. 101 from Blagoevgrad) satellites have not been found (Plate $2 \mathrm{~B}$ ). The chromosome number $2 n=18$ registered for the Bulgarian populations of $D$. botrys confirms the familiar reference data (MULLIGAN 1961; MEHRA and MALIK 1963; KeENER 1970; BASSET and CROMPTON 1971, 1982; Uotila 1973; QueIRos 1975, Schwarzová 1978b, 1980, 1993; DvořáK et al. 1980; KHATOON and Ali 1993; LOMONOSOVA et al. 2003).

The tetraploid chromosome number $2 n=32$ has been found for Dysphania ambrosioides. The same result has been found in various regions in the geographical area of the species (LORZ 1937; Woroschilov 1942; KAWATANI and OHNO 1950; RAGHAVAN and ARORA 1958; Mehra and Malik 1963; Sharma and Dey 1967; Murín and Ferakova 1974; QueIros 1975; Schwarzová 1978b, 1986; Silvestre 1984; Dvoř́́K 1989; KHATOON and Ali 1993; AL-TURKI et al. 1999, 2000). For the three studied populations, differences were recorded in the karyotype structure. In the two populations from the Thracian lowland (No. 32 from Plovdiv and No. 104 from an island on the Maritsa River) the karyotype consists of $2 n=20 \mathrm{~m}+12 \mathrm{sm}=32$ chromosomes (Plate 2C). For the population from the Danubian plain (No. 201 from the island of Milka) a karyotype of $2 n=4 \mathrm{~m}+28 \mathrm{sm}=32$ chromosomes has been established (Plate 2D).

The highest chromosome number $2 n=36$ is registered in the populations of $D$. multifida (Tab. 1). The same number was reported by MARKOVA (1968) for another Bulgarian population of the species from the village of Koshava in the Danubian plain. The data from the other parts of its habitat are for $2 n=32$ (KAWATANI and OHNO 1956; GIUSTI 1970; QueIRos 1975; GRANADO et al. 1988). In four of the studied populations (No. 39 from the island of Golyama Barzina, No. 108 from Parvomay, No. 105 from Kalofer, and No. 109 from Asenovgrad) the karyotype of $2 n=20 \mathrm{~m}+16 \mathrm{sm}=36$ chromosomes has been registered (Plate 3A). In the other three populations (No. 40 from Vidin, No. 106 from Ihtiman, No. 107 from Ivaylovgrad) the karyotype of $2 n=16 \mathrm{~m}+20 \mathrm{sm}=36$ chromosomes has been established (Plate 3B).
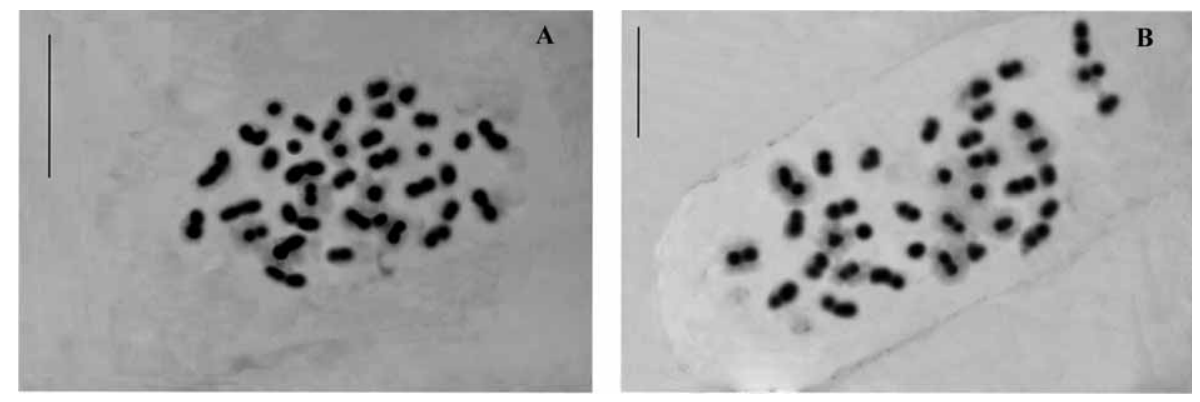

Plate 3. Microphotographs of the metaphase plate of Dysphania species: A - D. multifida (population № 105) $-2 n=36 ; \mathbf{B}-D$. multifida (№ 40) $-2 n=36$. - Scale bars $=10 \mu \mathrm{m}$.

\section{Morphology}

The intrapopulation variation was estimated on the basis of the coefficient of variation (Tabs. 2-4). The data show that in all populations vegetative have higher level of variability than generative traits. 
Grozeva N. H., Cvetanova Y. G.

Tab. 2. Mean (first line) and coefficient of variation in \% (second line) of Dysphania schraderiana (103), D. pumilio $(214,217,221)$, D. ambrosioides $(201,32,104)$ populations for each of the 24 observed characters

\begin{tabular}{|c|c|c|c|c|c|c|c|}
\hline & \multicolumn{7}{|c|}{ Populations } \\
\hline & 103 & 214 & 217 & 221 & 201 & 32 & 104 \\
\hline \multirow[t]{2}{*}{1} & 79.3 & 41.2 & 34.29 & 30.1 & 52.1 & 77.0 & 65.4 \\
\hline & 17.5 & 36.9 & 28.39 & 37.2 & 34.1 & 27.0 & 15.8 \\
\hline \multirow[t]{2}{*}{2} & 5.6 & 2.6 & 2.17 & 2.0 & 4.5 & 4.1 & 4.0 \\
\hline & 27.4 & 38.7 & 13.25 & 32.2 & 20.2 & 22.9 & 20.3 \\
\hline \multirow[t]{2}{*}{3} & 3.5 & 1.3 & 1.24 & 1.2 & 1.1 & 1.2 & 1.1 \\
\hline & 23.4 & 28.3 & 22.41 & 31.3 & 25.2 & 21.6 & 21.3 \\
\hline \multirow[t]{2}{*}{4} & 1.6 & 2.3 & 1.73 & 1.3 & 4.5 & 3.7 & 3.8 \\
\hline & 12.1 & 38.2 & 17.82 & 16.8 & 27.3 & 25.7 & 24.3 \\
\hline \multirow[t]{2}{*}{5} & 1.5 & 1.4 & 1.39 & 1.3 & 0.6 & 0.54 & 0.3 \\
\hline & 13.0 & 42.3 & 47.3 & 32.3 & 33.3 & 37.9 & 13.6 \\
\hline \multirow[t]{2}{*}{6} & 3.0 & 0.9 & 0.84 & 0.7 & 1.9 & 2.0 & 1.8 \\
\hline & 8.6 & 32.1 & 11.97 & 23.9 & 23.7 & 30.4 & 18.4 \\
\hline \multirow[t]{2}{*}{7} & 1.4 & 0.5 & 0.39 & 0.3 & 0.4 & 0.5 & 0.4 \\
\hline & 11.3 & 27.4 & 18.18 & 23.9 & 23.6 & 36.2 & 15.9 \\
\hline \multirow{2}{*}{8} & 2.2 & 1.5 & 1.52 & 1.4 & 5.8 & 4.5 & 5.1 \\
\hline & 6.9 & 44.2 & 21.34 & 19.8 & 25.2 & 29.0 & 21.0 \\
\hline \multirow[t]{2}{*}{9} & 0.3 & 0.5 & 0.53 & 0.6 & 0.4 & 0.3 & 0.1 \\
\hline & 26.2 & 35.2 & 12.30 & 14.6 & 32.7 & 35.8 & 23.0 \\
\hline \multirow[t]{2}{*}{10} & 50.9 & 21.7 & 19.94 & 15.1 & 29.6 & 45.4 & 40.8 \\
\hline & 22.2 & 24.3 & 38.61 & 32.2 & 46.9 & 40.8 & 25.2 \\
\hline \multirow[t]{2}{*}{11} & 0.4 & 0.0 & 0.0 & 0.0 & 0.0 & 0.1 & 0.1 \\
\hline & 30.6 & 0.0 & 0.0 & 0.0 & 23.4 & 28.3 & 31.0 \\
\hline \multirow[t]{2}{*}{12} & 0.8 & 0.8 & 0.75 & 0.7 & 1.0 & 1.0 & 1.1 \\
\hline & 15.0 & 15.4 & 12.34 & 11.3 & 3.4 & 5.2 & 5.9 \\
\hline \multirow[t]{2}{*}{13} & 0.8 & 0.8 & 0.85 & 0.9 & 1.0 & 1.0 & 1.1 \\
\hline & 10.2 & 18.5 & 11.25 & 9.5 & 5.7 & 5.2 & 8.1 \\
\hline \multirow[t]{2}{*}{14} & 0.5 & 0.4 & 0.37 & 0.3 & 0.6 & 0.6 & 0.6 \\
\hline & 16.1 & 12.3 & 18.42 & 14.2 & 9.3 & 8.9 & 0.0 \\
\hline \multirow[t]{2}{*}{15} & 0.8 & 0.8 & 0.75 & 0.8 & 1.0 & 1.0 & 1.0 \\
\hline & 7.2 & 9.4 & 9.40 & 8.4 & 4.7 & 9.0 & 6.4 \\
\hline \multirow[t]{2}{*}{16} & 0.5 & 0.4 & 0.31 & 0.3 & 0.5 & 0.6 & 0.6 \\
\hline & 11.9 & 11.5 & 9.72 & 9.3 & 7.0 & 8.6 & 7.2 \\
\hline \multirow[t]{2}{*}{17} & 0.8 & 0.8 & 0.78 & 0.72 & 0.7 & 0.5 & 0.6 \\
\hline & 16.7 & 4.9 & 4.95 & 5.2 & 16.3 & 13.7 & 6.3 \\
\hline \multirow[t]{2}{*}{18} & 0.7 & 0.7 & 0.67 & 0.7 & 0.6 & 0.5 & 0.6 \\
\hline & 11.6 & 7.5 & 7.50 & 5.6 & 12.6 & 8.9 & 4.1 \\
\hline \multirow[t]{2}{*}{19} & 1.1 & 1.2 & 1.24 & 1.3 & 1.1 & 1.0 & 1.0 \\
\hline & 12.0 & 4.8 & 4.35 & 5.8 & 14.0 & 13.0 & 5.1 \\
\hline \multirow[t]{2}{*}{20} & 0.4 & 0.2 & 0.34 & 0.4 & 0.4 & 0.3 & 0.4 \\
\hline & 12.5 & 6.8 & 17.62 & 13.3 & 12.1 & 10.9 & 5.1 \\
\hline
\end{tabular}


Tab. 2. - continued

\begin{tabular}{rrrlrrrr}
\hline & \multicolumn{7}{c}{ Populations } \\
\hline \multirow{2}{*}{21} & 103 & 214 & 217 & 221 & \multicolumn{1}{c}{201} & \multicolumn{1}{c}{32} & 104 \\
\hline \multirow{2}{*}{23} & 0.9 & 0.8 & 0.84 & 0.9 & 0.8 & 0.6 & 0.7 \\
& 13.3 & 7.5 & 7.5 & 8.4 & 12.8 & 10.9 & 5.0 \\
& 0.9 & 0.8 & 0.78 & 0.8 & 0.7 & 0.6 & 0.6 \\
& 13.3 & 8.4 & 8.44 & 5.5 & 11.6 & 8.0 & 0.0 \\
& 1.0 & 1.3 & 1.36 & 1.1 & 1.2 & 1.0 & 1.2 \\
& 10.9 & 7.3 & 7.36 & 6.8 & 8.7 & 13.0 & 5.1 \\
& 0.6 & 41.2 & 34.29 & 30.1 & 0.4 & 0.4 & 0.4 \\
& 9.8 & 36.9 & 28.39 & 37.2 & 8.4 & 12.1 & 8.4 \\
\hline
\end{tabular}

Tab. 3. Mean (first line) and coefficient of variation in \% (second line) of Dysphania botrys populations for each of the 24 observed characters

\begin{tabular}{rrrrrrrrrrrrr}
\hline & & & & & \multicolumn{1}{c}{ Populations } & & & & & & \\
& 29 & 97 & 100 & 35 & 102 & 99 & 98 & 101 & 53 & 30 & 34 & 45 \\
\hline 1 & 44.3 & 51.4 & 48.2 & 46.0 & 51.7 & 40.6 & 52.4 & 59.3 & 43.9 & 45.5 & 47.9 & 24.7 \\
& 24.3 & 25.2 & 17.5 & 20.2 & 20.8 & 40.2 & 12.4 & 16.1 & 27.5 & 27.6 & 14.4 & 44.2 \\
2 & 2.4 & 3.7 & 4.0 & 2.2 & 3.3 & 3.7 & 4.3 & 3.1 & 3.3 & 2.1 & 3.0 & 2.9 \\
& 30.4 & 38.5 & 40.3 & 12.9 & 41.4 & 34.1 & 28.8 & 28.2 & 40.6 & 24.4 & 47.9 & 46.0 \\
3 & 1.4 & 1.6 & 0.9 & 0.9 & 1.4 & 1.7 & 2.0 & 1.5 & 1.7 & 1.2 & 1.6 & 1.4 \\
& 26.5 & 44.6 & 19.4 & 19.8 & 39.7 & 44.6 & 27.5 & 26.8 & 40.8 & 24.4 & 41.8 & 43.0 \\
4 & 2.3 & 2.5 & 4.7 & 3.2 & 2.4 & 2.4 & 2.2 & 2.1 & 1.9 & 1.6 & 1.8 & 2.2 \\
& 35.4 & 31.7 & 29.2 & 39.7 & 32.5 & 21.3 & 27.5 & 26.2 & 24.1 & 22.6 & 20.3 & 15.1 \\
5 & 1.1 & 1.2 & 1.5 & 1.5 & 1.2 & 0.6 & 1.2 & 1.3 & 1.4 & 1.4 & 1.3 & 0.6 \\
& 37.3 & 26.8 & 17.5 & 17.5 & 37.4 & 32.5 & 20.9 & 26.5 & 28.4 & 23.1 & 19.6 & 32.2 \\
6 & 0.8 & 0.8 & 0.7 & 0.7 & 0.8 & 0.6 & 0.8 & 0.7 & 0.7 & 0.7 & 0.7 & 0.6 \\
& 26.1 & 29.4 & 12.8 & 11.7 & 28.7 & 27.1 & 12.9 & 17.0 & 11.7 & 16.0 & 14.6 & 31.5 \\
7 & 0.3 & 0.3 & 0.4 & 0.3 & 0.4 & 0.4 & 0.3 & 0.3 & 0.3 & 0.3 & 0.3 & 0.3 \\
& 18.2 & 30.8 & 14.4 & 11.4 & 23.0 & 24.1 & 11.0 & 26.5 & 22.9 & 21.9 & 16.5 & 39.0 \\
8 & 2.7 & 2.7 & 2.0 & 2.2 & 2.3 & 1.8 & 2.4 & 2.7 & 2.5 & 2.7 & 2.6 & 2.1 \\
& 39.2 & 40.2 & 19.6 & 10.9 & 38.5 & 22.5 & 16.6 & 18.3 & 17.7 & 17.2 & 24.6 & 23.9 \\
9 & 0.9 & 0.4 & 0.0 & 0.0 & 0.1 & 0.4 & 0.2 & 0.1 & 0.5 & 0.1 & 0.9 & 0.4 \\
& 29.5 & 45.8 & 39.3 & 40.4 & 49.5 & 47.8 & 46.6 & 55.1 & 11.3 & 46.3 & 49.6 & 47.0 \\
10 & 25.6 & 25.4 & 24.9 & 24.9 & 25.6 & 19.0 & 40.5 & 46.7 & 34.5 & 25.6 & 40.2 & 19.7 \\
& 34.7 & 35.3 & 18.9 & 56.6 & 34.7 & 35.8 & 16.6 & 8.8 & 30.6 & 34.7 & 17.0 & 38.7 \\
11 & 0.2 & 0.7 & 0.2 & 0.2 & 0.2 & 0.3 & 0.7 & 0.2 & 0.7 & 0.2 & 0.3 & 0.3 \\
& 40.1 & 43.3 & 51.6 & 51.6 & 34.5 & 15.8 & 43.2 & 45.9 & 43.2 & 43.0 & 35.6 & 15.6 \\
12 & 0.9 & 0.9 & 1.1 & 1.1 & 0.9 & 0.9 & 1.0 & 1.0 & 1.1 & 1.0 & 1.0 & 0.9 \\
& 8.6 & 8.6 & 4.9 & 4.9 & 8.5 & 9.7 & 4.8 & 4.9 & 5.0 & 4.8 & 4.8 & 9.5 \\
13 & 0.9 & 0.9 & 1.1 & 1.1 & 0.9 & 0.9 & 0.8 & 0.8 & 0.7 & 0.8 & 0.8 & 0.8 \\
& 12.0 & 10.4 & 7.2 & 7.2 & 11.5 & 14.9 & 6.8 & 10.2 & 6.7 & 14.3 & 6.8 & 13.8 \\
& 0.3 & 0.4 & 0.4 & 0.3 & 0.4 & 0.3 & 0.3 & 0.3 & 0.4 & 0.3 & 0.3 & 0.3 \\
& 12.7 & 15.8 & 16.4 & 14.1 & 17.3 & 16.6 & 14.1 & 9.8 & 14.5 & 10.5 & 13.8 & 13.3 \\
\hline \multirow{4}{*}{50}
\end{tabular}


Grozeva N. H., Cvetanova Y. G.

Tab. 3. - continued

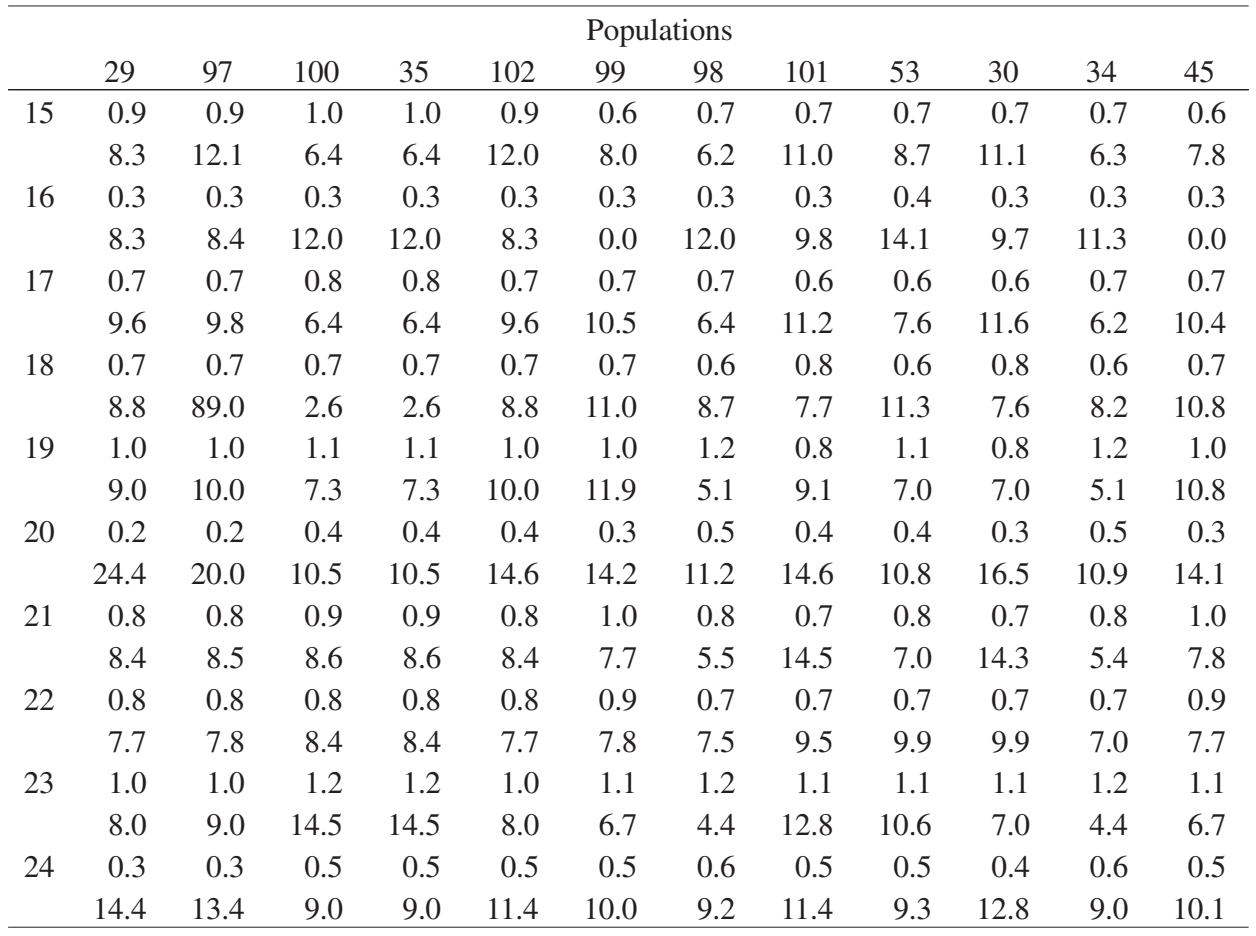

Tab. 4. Mean (first line) and coefficient of variation in \% (second line) of Dysphania multifida populations for each of the 24 observed characters

\begin{tabular}{|c|c|c|c|c|c|c|c|}
\hline & \multicolumn{7}{|c|}{ Populations } \\
\hline & 103 & 214 & 217 & 221 & 201 & 32 & 104 \\
\hline \multirow[t]{2}{*}{1} & 51.2 & 90.9 & 73.4 & 88.6 & 83.6 & 54.2 & 65.5 \\
\hline & 21.9 & 15.6 & 30.6 & 24.6 & 24.9 & 29.1 & 32.5 \\
\hline \multirow[t]{2}{*}{2} & 1.3 & 1.3 & 1.4 & 1.6 & 1.4 & 1.2 & 1.2 \\
\hline & 32.9 & 11.9 & 24.7 & 15.2 & 20.8 & 32.1 & 25.7 \\
\hline \multirow[t]{2}{*}{3} & 0.5 & 0.6 & 0.6 & 0.8 & 0.7 & 0.6 & 0.6 \\
\hline & 35.5 & 18.5 & 23.8 & 9.3 & 19.9 & 35.7 & 32.8 \\
\hline \multirow[t]{2}{*}{4} & 2.6 & 2.3 & 2.5 & 1.9 & 2.1 & 2.5 & 2.1 \\
\hline & 39.1 & 23.4 & 26.5 & 20.1 & 21.1 & 51.4 & 44.0 \\
\hline \multirow[t]{2}{*}{5} & 0.4 & 0.4 & 0.4 & 0.5 & 0.4 & 0.2 & 0.4 \\
\hline & 20.9 & 21.1 & 18.7 & 11.7 & 17.0 & 22.6 & 20.7 \\
\hline \multirow[t]{2}{*}{6} & 0.5 & 0.8 & 0.7 & 0.7 & 0.8 & 0.5 & 0.6 \\
\hline & 31.5 & 17.2 & 23.6 & 10.6 & 15.3 & 36.6 & 32.4 \\
\hline \multirow[t]{2}{*}{7} & 0.3 & 0.4 & 0.4 & 0.5 & 0.4 & 0.3 & 0.3 \\
\hline & 35.2 & 20.5 & 17.6 & 11.0 & 17.0 & 21.3 & 37.8 \\
\hline \multirow[t]{2}{*}{8} & 1.9 & 2.3 & 1.8 & 1.6 & 1.8 & 1.9 & 1.9 \\
\hline & 22.2 & 20.4 & 28.8 & 15.1 & 20.2 & 26.3 & 23.9 \\
\hline
\end{tabular}


Tab. 4. - continued

\begin{tabular}{|c|c|c|c|c|c|c|c|}
\hline & \multicolumn{7}{|c|}{ Populations } \\
\hline & 103 & 214 & 217 & 221 & 201 & 32 & 104 \\
\hline \multirow[t]{2}{*}{9} & 0.2 & 0.2 & 0.3 & 0.3 & 0.3 & 0.2 & 0.3 \\
\hline & 19.3 & 20.3 & 19.1 & 20.7 & 22.1 & 29.9 & 22.9 \\
\hline \multirow[t]{2}{*}{10} & 36.9 & 73.1 & 49.3 & 64.6 & 63.3 & 39.6 & 43.9 \\
\hline & 28.8 & 19.8 & 38.8 & 15.8 & 22.9 & 33.5 & 33.0 \\
\hline \multirow[t]{2}{*}{11} & 0.1 & 0.3 & 0.2 & 0.3 & 0.2 & 0.1 & 0.1 \\
\hline & 54.1 & 43.7 & 47.3 & 42.6 & 41.7 & 40.1 & 44.7 \\
\hline \multirow[t]{2}{*}{12} & 1.2 & 1.2 & 1.1 & 1.0 & 1.1 & 1.2 & 1.2 \\
\hline & 4.9 & 16.4 & 14.3 & 10.3 & 10.2 & 8.8 & 9.6 \\
\hline \multirow[t]{2}{*}{13} & 1.0 & 1.0 & 1.0 & 1.1 & 1.1 & 1.0 & 1.0 \\
\hline & 6.6 & 12.6 & 10.6 & 8.4 & 7.8 & 8.9 & 11.4 \\
\hline \multirow[t]{2}{*}{14} & 0.4 & 0.4 & 0.4 & 0.5 & 0.4 & 0.4 & 0.4 \\
\hline & 13.8 & 9.3 & 16.9 & 12.2 & 7.8 & 13.4 & 16.9 \\
\hline \multirow[t]{2}{*}{15} & 0.0 & 0.0 & 0.0 & 0.0 & 0.0 & 0.0 & 0.0 \\
\hline & 0.0 & 0.0 & 0.0 & 0.0 & 0.0 & 0.0 & 0.0 \\
\hline \multirow[t]{2}{*}{16} & 0.0 & 0.0 & 0.0 & 0.0 & 0.0 & 0.0 & 0.0 \\
\hline & 0.0 & 0.0 & 0.0 & 0.0 & 0.0 & 0.0 & 0.0 \\
\hline \multirow[t]{2}{*}{17} & 0.8 & 0.9 & 0.8 & 0.8 & 0.8 & 0.8 & 0.8 \\
\hline & 7.2 & 4.3 & 7.8 & 8.1 & 7.8 & 5.9 & 7.3 \\
\hline \multirow[t]{2}{*}{18} & 0.8 & 0.8 & 0.8 & 0.9 & 0.8 & 0.8 & 0.8 \\
\hline & 8.2 & 5.4 & 6.6 & 6.0 & 5.6 & 8.9 & 7.7 \\
\hline \multirow[t]{2}{*}{19} & 1.1 & 1.1 & 1.1 & 0.9 & 1.0 & 1.1 & 1.0 \\
\hline & 8.5 & 7.6 & 6.5 & 11.1 & 11.0 & 5.2 & 8.7 \\
\hline \multirow[t]{2}{*}{20} & 0.3 & 0.3 & 0.3 & 0.4 & 0.3 & 0.3 & 0.3 \\
\hline & 18.9 & 0.0 & 16.0 & 14.7 & 14.6 & 20.9 & 13.2 \\
\hline \multirow[t]{2}{*}{21} & 0.9 & 1.0 & 0.9 & 1.0 & 1.0 & 0.9 & 0.9 \\
\hline & 6.4 & 5.3 & 7.1 & 6.5 & 4.6 & 5.8 & 6.4 \\
\hline \multirow[t]{2}{*}{22} & 0.9 & 0.9 & 0.9 & 0.9 & 0.9 & 0.9 & 0.9 \\
\hline & 7.2 & 0.0 & 8.1 & 8.9 & 8.1 & 7.6 & 8.3 \\
\hline \multirow[t]{2}{*}{23} & 1.1 & 1.0 & 1.1 & 1.1 & 1.1 & 1.0 & 1.0 \\
\hline & 7.5 & 8.0 & 5.7 & 8.9 & 10.0 & 9.6 & 6.7 \\
\hline \multirow[t]{2}{*}{24} & 0.4 & 0.4 & 0.4 & 0.4 & 0.4 & 0.4 & 0.4 \\
\hline & 7.8 & 0.0 & 7.8 & 0.0 & 0.0 & 15.6 & 5.2 \\
\hline
\end{tabular}

The results of the ANOVA (Tab. 5) demonstrate that in all species, interpopulation variability is dominant in total variability.

The interpopulation variation was evaluated in Dysphania botrys, D. pumilio, D. ambrosioides and $D$. multifida, as $D$. schraderiana was investigated in a single population. The values of Euclidean distances (Ed) varied within different limits in the individual taxa (Tabs. 6-9)

The UPGA cluster analysis based on the morphological pairwise similarities (Euclidean distances between population centroids) enabled us to detect two main clusters A and B 
Grozeva N. H., Cvetanova Y. G.

Tab. 5. Percentage of the interpopulation variation in the overall morphological variation for each character in Dysphania

\begin{tabular}{ccccc}
\hline \multirow{2}{*}{ Character № } & \multicolumn{4}{c}{ Species } \\
& D. botrys & D. pumilio & D. ambrosioides & D. multifida \\
\hline 1 & 37.7 & 30.1 & 66.0 & 43.2 \\
2 & 29.2 & 45.7 & 47.7 & 29.2 \\
3 & 65.1 & 47.6 & 43.4 & 64.5 \\
4 & 46.4 & 70.9 & 38.1 & 49.9 \\
5 & 69.1 & 32.2 & 48.0 & 61.1 \\
6 & 61.8 & 51.3 & 31.1 & 37.0 \\
7 & 66.5 & 46.1 & 68.0 & 53.9 \\
8 & 67.7 & 55.8 & 40.2 & 57.3 \\
9 & 45.1 & 66.3 & 74.6 & 57.9 \\
10 & 76.6 & 65.3 & 71.1 & 48.7 \\
11 & 69.3 & 0.0 & 59.4 & 50.6 \\
12 & 52.1 & 55.7 & 62.3 & 55.7 \\
13 & 66.5 & 50.9 & 62.9 & 52.9 \\
14 & 55.7 & 59.0 & 64.9 & 38.2 \\
15 & 78.9 & 52.7 & 55.7 & 0.0 \\
16 & 33.1 & 56.8 & 59.9 & 0.0 \\
17 & 41.6 & 59.5 & 85.1 & 61.4 \\
18 & 57.0 & 56.2 & 94.0 & 63.0 \\
19 & 57.0 & 49.3 & 65.1 & 52.4 \\
20 & 76.8 & 52.8 & 78.7 & 61.5 \\
21 & 52.5 & 54.9 & 91.5 & 58.7 \\
22 & 66.4 & 52.2 & 91.6 & 55.2 \\
23 & 64.0 & 50.1 & 56.6 & 68.4 \\
24 & 61.7 & 50.6 & 0.0 & 52.1 \\
\hline
\end{tabular}

Tab. 6. Values of Euclidean distances between the pairs of populations of $D$. pumilio based on 24 characters.

\begin{tabular}{crc}
\hline $\begin{array}{l}\text { Number of } \\
\text { population }\end{array}$ & 214 & 217 \\
\hline 217 & 54 & \\
221 & 111 & 57 \\
\hline
\end{tabular}

Tab. 7. Values of Euclidean distances between pairs of populations of $D$. ambrosioides based on 24 characters.

\begin{tabular}{ccc}
\hline $\begin{array}{c}\text { Number of } \\
\text { population }\end{array}$ & 32 & 104 \\
\hline 104 & 125 & \\
201 & 295 & 194 \\
\hline
\end{tabular}

(Fig. 1). Cluster A consists of two subclusters $A_{1}$ and $A_{2}$. The populations of Dysphania multifida form the subcluster $\mathrm{A}_{1}$, while the populations of $D$. botrys, $D$. pumilio and $D$. schraderiana were grouped by their own species in the subcluster $\mathrm{A}_{2}$. Cluster B contains the populations of D. ambrosioides. 
Tab. 8. Values of Euclidean distances between the pairs of populations within Dysphania multifida based on 24 characters

\begin{tabular}{crrrrrr}
\hline $\begin{array}{c}\text { Number of } \\
\text { population }\end{array}$ & 39 & 40 & 105 & 106 & 107 & 108 \\
\hline 40 & 537 & & & & & \\
105 & 254 & 296 & & & & \\
106 & 465 & 122 & 216 & & & \\
107 & 418 & 89 & 173 & 51 & & \\
108 & 40 & 497 & 215 & 425 & 378 & \\
109 & 158 & 388 & 96 & 310 & 266 & 121 \\
\hline
\end{tabular}

Tab. 9. Values of Euclidean distances between the pairs of populations of Dysphania botrys based on 24 characters

\begin{tabular}{crrrrrrrrrrr}
\hline $\begin{array}{c}\text { Number of } \\
\text { Population }\end{array}$ & 29 & 97 & 100 & 35 & 102 & 99 & 98 & 101 & 53 & 30 & 34 \\
\hline 97 & 72 & & & & & & & & & & \\
100 & 43 & 34 & & & & & & & & & \\
35 & 22 & 59 & 31 & & & & & & & & \\
102 & 74 & 5 & 37 & 60 & & & & & & & \\
99 & 77 & 126 & 97 & 83 & 129 & & & & & & \\
98 & 171 & 152 & 162 & 171 & 150 & 245 & & & & & \\
101 & 296 & 265 & 273 & 283 & 266 & 267 & 410 & & & & \\
53 & 90 & 119 & 106 & 100 & 119 & 158 & 105 & 378 & & & \\
30 & 13 & 62 & 34 & 15 & 63 & 84 & 166 & 290 & 92 & & \\
34 & 151 & 152 & 153 & 155 & 151 & 224 & 47 & 417 & 69 & 148 & \\
45 & 205 & 274 & 241 & 220 & 276 & 160 & 347 & 398 & 243 & 216 & 309 \\
\hline
\end{tabular}

The results from the SDA are presented in table 10. The first three discriminant (canonical) functions account for over $98 \%$ of the total variance. Overall, the discrimination between species is highly significant (Wilks' Lambda $=0.00026, \mathrm{~F}=567.01, \mathrm{p}<0.001$ ). Wilks' Lambda is the standard statistic that has been used to denote the statistical significance of the discriminatory power of the current model. The closer to 0 the Wilks' Lambda, the greater is the contribution of the model to the overall discrimination. The Partial Wilks' Lambda values in the first column (Tab. 10) present the unique contribution of each character in the model to the discrimination between species. The lower the value in this column, the greater is the unique discriminatory power of the respective character. All discriminant functions and variables in the model were highly significant $(\mathrm{p}<0.001)$

The standardized discriminant function coefficients determine the unique contribution of each character to the discriminant functions D1-D3. The first discriminant function which provides the most overall discrimination between the five species is loaded most heavily by the width and the length of perianth lobes in the female flower, and the length of perianth lobes in the bisexual flower. The second discriminant function seems to be marked 


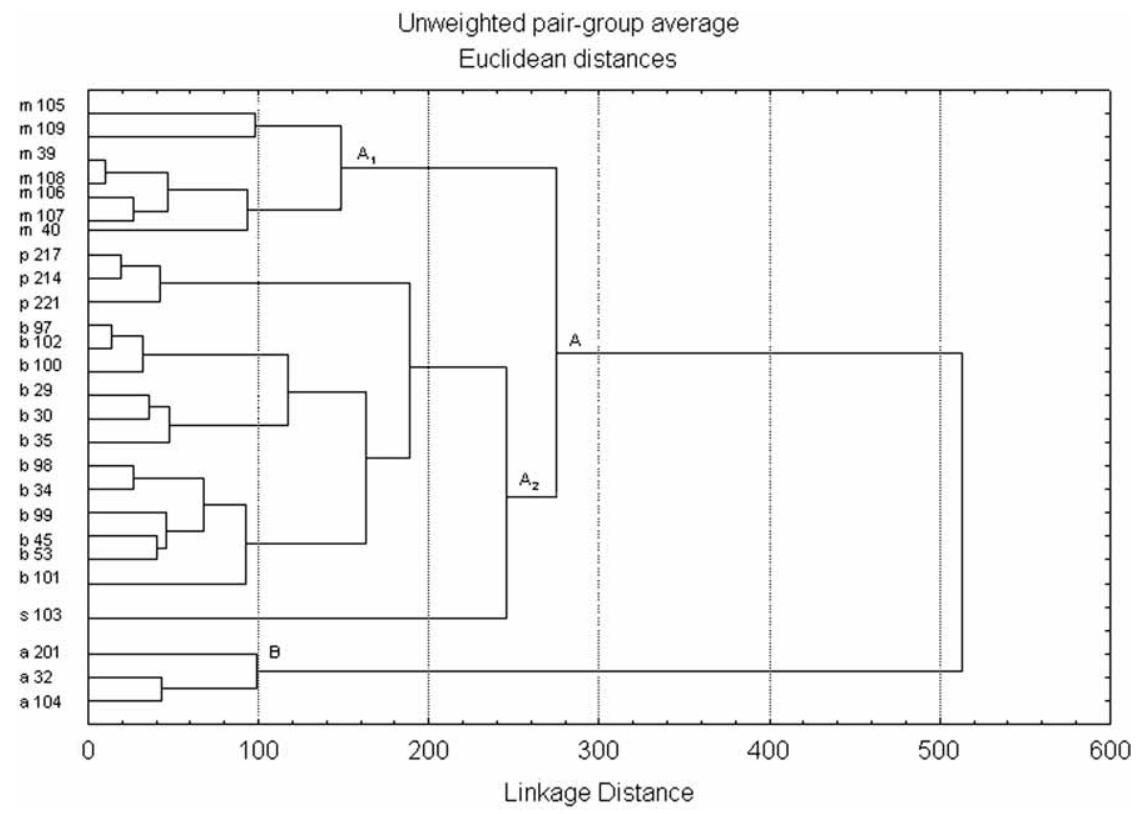

Fig. 1. Dendrogram of the cluster analysis of the genus Dysphania: № $105-40=D$. multifida (m); № $217-221=D$. pumilio $(\mathrm{p})$; № 97-101 $=$ D. botrys $(\mathrm{b})$; № $103=D$. schraderiana (s); № $201-104=$ D. ambrosioides (a)

Tab. 10. The sequence of the ten most significant characters for discrimination between five species of genus Dysphania obtained by SDA and corresponding values of Partial Wilks' Lambda, F-values and standardized coefficients for discriminant functions $D_{1}-D_{3}$. The eigenvalues, cumulative percentage of the variance explained by the first three roots are presented in the bottom two rows.

\begin{tabular}{|c|c|c|c|c|c|}
\hline Characters & F-remove & $\begin{array}{l}\text { Wilks' } \\
\text { Lambda }\end{array}$ & $D_{1}$ & $\mathrm{D}_{2}$ & $\mathrm{D}_{3}$ \\
\hline $\begin{array}{l}\mathrm{x}_{16 .} \text {. Width of perianth lobes in } \\
\text { female flower }\end{array}$ & 307.0124 & 0.000674 & -0.75486 & -0.096454 & -0.255158 \\
\hline$x_{7}$. Width of upper leaf & 169.4663 & 0.000488 & -0.03937 & -0.384323 & 0.715588 \\
\hline $\mathrm{x}_{6}$. Length of upper leaf & 137.8743 & 0.000445 & -0.12250 & -0.666162 & -0.372245 \\
\hline $\begin{array}{l}\mathrm{x}_{15} \text {. Length of perianth lobes in } \\
\text { female flower }\end{array}$ & 137.6188 & 0.000445 & -0.71658 & 0.390183 & 0.069843 \\
\hline $\begin{array}{l}\mathrm{x}_{13} \text {. Length of perianth lobes in } \\
\text { bisexual flower }\end{array}$ & 72.0499 & 0.000356 & 0.47251 & -0.214634 & -0.386907 \\
\hline $\mathrm{x}_{24}$. Thickness of fruit & 54.0739 & 0.000332 & -0.15462 & -0.220721 & 0.206799 \\
\hline $\mathrm{x}_{11}$. Length of flower petiole & 85.6233 & 0.000374 & -0.01660 & -0.107996 & 0.471146 \\
\hline $\mathrm{x}_{5}$. Length of basal leaf petiole & 62.8143 & 0.000343 & 0.10170 & 0.156215 & 0.544039 \\
\hline $\mathrm{x}_{12 .}$. Diameter of flower & 45.4535 & 0.000320 & 0.28564 & -0.306588 & -0.234661 \\
\hline $\mathrm{x}_{9}$. Llength of upper leaf petiole & 37.9717 & 0.000310 & 0.10216 & -0.273894 & -0.373920 \\
\hline Eigenvalues & & & 41.98092 & 7.680871 & 4.657472 \\
\hline Cumulative Prop. of Variance & & & 0.76118 & 0.900440 & 0.984887 \\
\hline
\end{tabular}




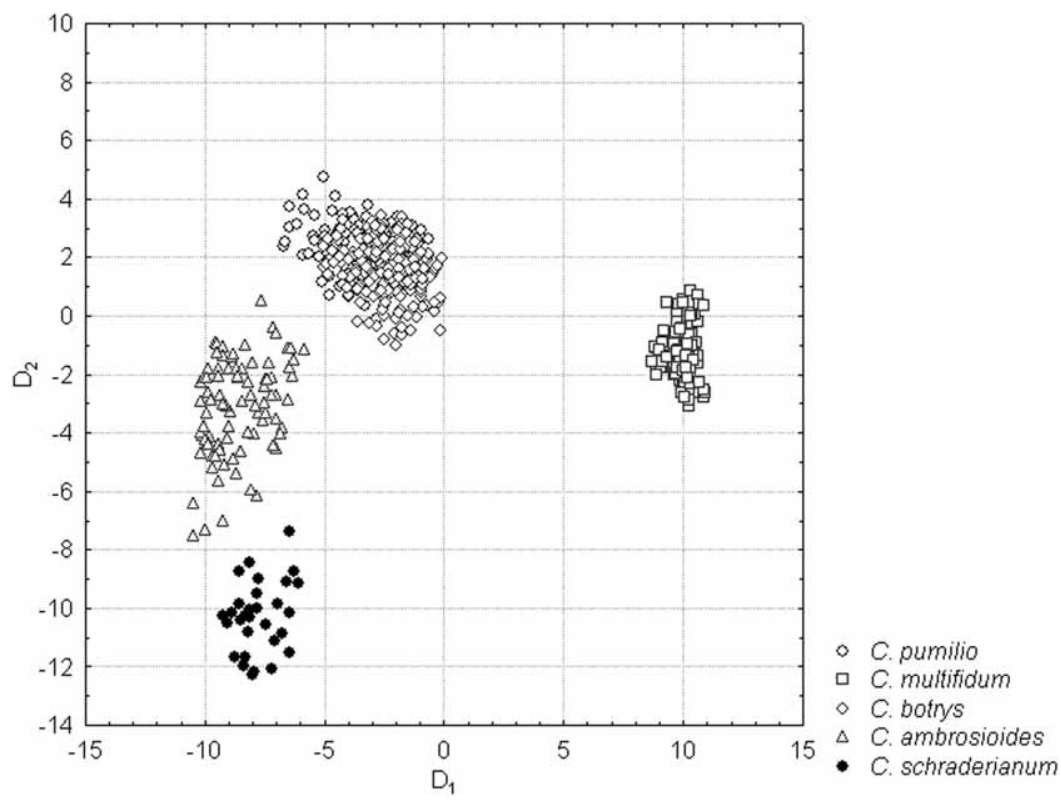

Fig. 2. Scatter plot of scores of the first two canonical roots $D_{1}$ and $D_{2}$ for the individuals of the five Dysphania species

mostly by the length of the upper leaf, while the width of the upper leaf, length of flower petiole and length of basal leaf petiole contribute mostly to the third discriminant function.

The populations of $D$. multifida, D. ambrossoides and D. schraderiana have been classified $100 \%$ correctly on the fourth step of the SDA while the populations of the other two species D. botrys and D. pumilio were classified with $98.46 \%$ and $92.22 \%$ correctness, respectively, on the tenth step.

The scatter plot of the canonical scores of the individuals from the five species on the first two discriminant dimensions (Fig. 2) enabled us to determine the nature of the discrimination for the first two discriminant roots. The first discriminant function mostly discriminates between $D$. multifida and the four other species. Clearly, the populations of $D$. multifida are plotted much further to the right of the central line. The second function seems to provide some discrimination between the species of $D$. ambrosioides, $D$. schraderiana and $D$. multifida (which mostly show negative values for the second canonical function) and the other two species D. botrys and D. pumilio (which have mostly positive values).

\section{Discussion}

The results of the present complete population research of genus Dysphania confirm the data from our previous research (GROZEVA and CVETANOVA 2008, 2011) on the species from family Chenopodiaceae, namely that vegetative traits are more variable than generative ones (Tabs. 2-4). The lamina size of the lower leaves, petiole length of upper or lower leaves and stem height are the most variable traits in almost all populations. The characteristics of the seed dimensions (i.e., length, width and ratio between them) are some of the most con- 
servative traits. No variability has been recorded for the trait length of female flower perianth in two of the populations of D. botrys, No. 45 from Tselina and No. 99 from Troyan. The high variability of stem height and leaf characters of the species from Chenopodium senso lato, and the lack of any considerable variability in seed characters have been acknowledged by researchers of the genus from various parts of the world (KowAL 1953, ENGSTRAND and Gustafsson 1972-1974, ReYNOLDS and CRAUfORD 1980, BASSET and Crompton 1982, DostáleK et al. 1990, Al-Turki and GHAFOOR 1996, Uotila 2001).

The main source of phenotype variation in all species is the interpopulation variation. The interpopulation differences were fewer and varied within much narrower limits in $D$. pumilio (Tab. 6). That is associated with the small differences between population habitats (Tab. 1) as well as with the lack of karyotype variability. Low variability level has been found among the populations of $D$. ambrosioides (Tab. 7), which relates to their similar habitats (sandy terrains in close proximity to a water basin) and similar altitudes (28-164 m). The recorded higher variability for the population from the Danubian plain than in the two populations from the Thracian lowland could be explained by either the differences in the soil and climatic conditions due to their geographic remoteness or the established karyotype differences.

Large interpopulation morphological variation was observed in D. multifida (Tab. 8) and the greatest differences have been recorded among populations with different karyotypes. Among populations with the same structure of the karyotypes, strong morphological similarity was recorded between populations with similar community components - No. 39 from the island of Golyama Barzina and No. 108 from Parvomay $(\mathrm{Ed}=40)$, where $D$. multifida dominates and cereal species are quite limited; No. 106 from Ihtiman and No. 107 from Ivaylovgrad $(\mathrm{Ed}=51)$, where cereal species are dominant. According to our observations, the established relationship is directly linked to the biological characteristics of the species. D. multifida is a perennial grass plant with a shallow root system and highly branched creeping stems. Its normal vegetative development needs space and efficient amount of water and food supplies in the topsoil, and these depend on the composition of the population and the existing relationships within it.

The largest interpopulation morphological variation was observed in Dysphania botrys (Tab. 9) which is in agreement with the diversity of ecological conditions, altitudes (0-748 $\mathrm{m}$ ), ecosystems (Tab. 1), as well as the constituted kariotype differences. The interpopulation differences within this species were the least between populations No. 97 from Pomorie, No. 102 from »Sinite kamani « Natural Park $(E d=5)$, and population No. 29 from the Varna lake and population No. 30 from Plovdiv $(\mathrm{Ed}=13)$, which have identical karyotype structures. High morphological similarity was found between population No. 35 from Belene and population No. 30 from Plovdiv $(\mathrm{Ed}=15)$, and population No. 29 from Varna Lake $(E d=22)$, which are with different karyotypes, although they have similar ecological conditions (altitudes up to $200 \mathrm{~m}$, flat terrain, close to a water basin with moist and sandy soil). Of all studied populations, the greatest differences are those from Tselina (population № 45) and Blagoevgrad (population № 101) where the species is a weed in a cultivated community. This is related to better soil conditions (i.e., food substances, moisture, and aeration), and weaker competition between the cultivated and weed plant than in the species' natural habitat. Regardless of the similar composition of communities, both populations differ significantly from each otehr, which relates to their appurtenance to two different and quite distant floristic regions and to the differences found in their karyotypes. 
The interpopulation variability is dominant within the total variability in all species (Tab. 5), which is due mainly to differences in ecological conditions and especially in the various plant communities to which they belong, as for the populations of $D$. ambrosioides, $D$. botrys and $D$. multifida karyological variability is also a factor. The less pronounced intrapopulation variability relates to the smaller number and area of the population, and the uniform ecological conditions within its borders.

After comparing all populations of the species in the genus according to the entire morphological set of quantitative traits (Fig. 1), the greatest similarity was found between the two diploid species $D$. botrys and $D$. pumilio. Quite similar to these two is the third diploid species $-D$. schraderiana. The three species also have similar ecological preferences. A certain morphological similarity was registered between the tetraploid D. multifida and the three diploid species, as well as between the tetraploid $D$. ambrosioides and all other species in the genus. Such morphological similarity among the five species was also noted by UotILA (2001) in Flora Nordica.

In spite of the high morphological similarity among the species, each one of them could be recognized correctly by certain characteristics (Fig. 2). The top ten significant features for differentiation of species, found by SDA were: width of perianth lobes in female flower, width of upper leaf, length of upper leaf, length of perianth lobes in female flower, length of perianth lobes in bisexual flower, thickness of fruit, length of flower petiole, length of basal leaf petiole, diameter of flower, length of upper leaf petiole (Tab. 10). The distinction between D. multifida, D. ambrosioides and D. schraderiana is based on differences in the quantitative traits, while such discrimination between $D$. botrys and $D$. pumilio is not always feasible. Due to the similarity in the greater part of the qualitative traits of these two species, qualitative characteristics should be used as well for their clear-cut distinction. D. pumilio could be distinguished easily from $D$. botrys as well as from the other species of the genus by its 4-5 pale green perianth leaves that harden and whiten at the fruit, as well as by reddish-brown to brown-black seeds with an imperceptible blunt edge. D. botrys was characterized by 5 yellowish-green perianth leaves that do not fully cover the fruit and do not harden and greyish-black seeds with an edge longitudinally split up in two. D. schraderiana differed by 4-5 perianth lobes with clearly defined serrated dorsal edge and black seeds with blunt edge. D. ambrosioides is easily recognizable by its five pale green perianth lobes with clearly defined non-serrated dorsal edge, connate up to $1 / 2$ of their length and brown seeds with blunt edge. D. multifida differed to the greatest degree from the other species with its five heavily furrowed perianth lobes, with no dorsal edge, connate along their entire length, that are preserved at the fruit and form a false pod and dark brown round seeds. Each species could also be identified by its typical indentation of the leaf lamina, however, that is not always a sure method since it is necessary to collect a greater number of whole plants in order to trace the lamina changeability of the lower, middle, and upper leaves.

The data from the present study confirm that polyploidy and diploidy are basic evolutionary mechanisms in genus Dysphania. In tracing the morphological variability, the tendency, known for Chenopodium senso lato, to reduce the sizes of the generative organs and the number of perianth lobes was established. The reduced size of perianth lobes and the decrease in their number facilitate anemophilous pollination. The retention of the perianth lobes at the fruit and the presence of glands on their surface is an adaptation to exozoochory. The nuts stick to animal fur and are carried as they move around, while the reduced size of 
the seeds favours their transportation at greater distances. Perianth lobes in some species have additional features securing reliable attachment: rough surface in $D$. multifida, pointed dorsal edge in D. ambrosioides, serrated dorsal edge in D. schraderiana). The lack of a dorsal edge on the perianth lobes of $D$. botrys and $D$. pumilio is perfectly compensated for by there being more glands on the entire surface of the first species, and on the upper parts of the second.

\section{Acknowledgements}

This study was supported by the Project Scientific Research Fund of Trakya University, Agriculture faculty (Project 2P/08). The authors are grateful to the anonymous reviewers for the valuable comments and suggestions.

\section{References}

Aellen, P., 1960: Chenopodium L. In: ReICHINGer, K. H., Hegis Illustrierte Flora von Mitteleuropa, 3.2., 569-664. Carl Hanser Verlag, Berlin - Hamburg.

Al-Turki, T. A., Ghafoor, A. 1996: The genus Chenopodium L. in Saudi Arabia. Feddes Repertorium 107, 189-208.

Al-Turki, T. A., Mehmood, S. F., Filfilan, S. A., 1999: IOPB chromosome data 15. Newsletter of International Organization of Plant Biosystematists (Pruhonice) 31, 10.

Al-Turki, T. A., Filfilan, S. A., Mehmood, S. F., 2000: A cytological study of flowering plants from Saudi Arabia. Willdenowia 30, 339-358.

Assyov, B., Petrova, A. (eds), 2006: Conspectus of the Bulgarian vascular flora. Distribution maps and floristic elements, 3 (In Bulgarian). BBF, Sofia.

Basset, I., Crompton, C., 1971: Reports. In: LÖVE, Å. (ed.), Chromosome number reports 34. Taxon 20, 785-797.

Basset, I. J., CRompton, C. W., 1982: The genus Chenopodium in Canada. Canadian Journal of Botany 60, 586-610.

Clements, S. E., Mosyakin, S. I., 2003: Dysphania R. Br. In: Editorial Committee (eds), Flora of North America north of Mexico, 4: Magnoliophyta: Caryophyllidae, 1, 267299. Oxford University Press, New York.

DostáleK, J. 1985: Determination of Chenopodium species of flora Czechoslovakia (in Czech). Zprávy České Botanické Společnosti 20, 161-177.

Dostálek, J., HejnỲ, S., HusÁk, Š., SchwarzovÁ, T., DvořÁK, F., 1990: Chenopodium L. In: HejnỲ, S., Slavík, B. (eds), Flora of the Czech Republic, 2 (in Czech), 223-265. Academia, Prague.

DVoŘÁK, F., 1989: Chromosome counts and chromosome morphology of some selected species. Scripta Facultatis Scientiarum Naturalium Universitatis Purkyn 19, 301-322.

DVOŘÁK, F., GRŰLl, F., KURKA, R., RŮŽIČKA, I., DADAKOVÁ, B., 1980: Reports. In: LÖVE, Á. (ed.), Chromosome number reports 68. Taxon 29, 533-547.

Engstrand, L., Gustafsson, M. 1972-1974: Drawings of Scandinavian plants. Chenopodium. Botaniska Notiser 125, 283-286; 126, 1-6, 135-141, 273-276, 393-397; 127, 1-6, 159-164, 291-296, 457-463. 
Giusti, L., 1970: Genus Chenopodium in Argentina. 1: Number of chromosomes (in German). Darwiniana 16, 98-105.

Granado, C., Luque, T., Pastor, J., 1988: Chromosome numbers from Spanish flora 551-555 (in Spanish), Lagascalia 15, 133-136.

GrIF, V. G., AGAPova, N., D., 1986: The methods of description of plant karyotypes (in Russian). Botanichesky Zhurnal 71, 550-553.

Grozeva, N., Cvetanova, Y. 2008: Variability among the populations of two Chenopodium genus species. Proceedings 7 Scientific Conference of Ekology and Health, Academic Publishing House of Agrarian University. Plovdiv, 135-140.

Grozeva, N., Cvetanova, Y. 2011: Chenopodium bonus-henricus (Perennial goosefoot) in Bulgaria. I. Population variability. Trakia Journal of Sciences 9, 1-7.

Grozeva, N., 2007: C. pumilio (Chenopodiaceae): a new species to the Bulgarian flora. Phytologia Balcanica 13, 331-334.

JAVOURKOVÁ-JAROLÍMOVÁ, V. 1992: Chenopodium pumilio R. Br. In: MĚsíčEK, J., JAVOURKOVÁ-JAROLÍMOVÁ,, V. List of chromosome numbers of the Czech vascular plants (in Czech), 75. Academia, Prague.

KaWATAni, T., Ohno, T., 1950: Chromosome numbers of genus Chenopodium: I. Chromosome number of Mexican tea (Ch. ambrosioides), American wormseed (Ch. ambrosioides L. var. altherminticum A Gray) and some allied species. Japanese Journal of Genetics $25,177-180$.

Kawatani, T., Ohno T., 1956: Chromosome numbers of genus Chenopodium. 2. Japanese Journal of Genetics 31, 15-17.

KeEner, C. 1970: Documented plant chromosome numbers 70. Sida Contributions to Botany 3, 533-536.

Khatoon, S., Ali S, I., 1993: Chromosome atlas of the angiosperms of Pakistan. Department of Botany, University of Karachi, Karachi.

KJELlmark, S., 1934: Some new chromosome numbers interpreted species (in German). Botaniska Notiser 117, 389-396.

KowAL, T. 1953: A key for the determination of the seeds of the genera Chenopodium and Atriplex (in Polish). Monographiae Botanicae 1, 87-163.

KÜHN, U. 1993: Chenopodiaceae. In: KuBITZKI, K. (ed.), The families and genera of vascular plants, 2, 253-281. Springer, Hamburg.

Lomonosova, M. N., S. A. Krasnikova, S. A., Krasnikov, A. A., Ebel, A. L., Rudaja, N. A., 2003: Chromosome numbers of the family members Chenopodiaceae representatives from Mongolia and Kazakhstan. Botanichesky Zhurnal 88, 113-115.

LoRZ, A., 1937: Cytological investigations on five Chenopodiaceous genera with special emphasis on chromosome morphology and somatic doubling in Spinacia. Cytologia 8, 241-276.

MARKova, İ., 1966: Chenopodium L. In: YORDANOV, D. (ed.), Flora of the People's Republic Bulgaria, 3, 527-541 (in Bulgarian). Aedibus Academiae Scientiarum Bulgaricae, Serdicae. 
Markova, M., 1968: Karyological study of Epimedium pubigerum (DC) Morren and Decne and Chenopodium multifidum L. Comptes Rendus de lộAcademie Bulgare des Sciences 21, 51-53.

Mehra, P., Malik, C., 1963: Cytology of some Indian Chenopodiaceae. Caryologia 16, 67-84.

MesíčEK, J., 1992: Chenopodium pumilio R. Br. In: MesíčEK, J., JavourkovÁ-JarolímovÁ, V., List of chromosome numbers of the Czech Vascular Plants (in Czech), 75. Academia, Prague.

Mosyakin, S. L., 1993: An outline of a system for Chenopodium L. (species of Europe, North and Central Asia). Ukrainsky Botanichny Zhurnal 50, 71-77.

Mosyakin, S. L., Clements, S. E., 1996: New infrageneric taxa and combination in Chenopodium L. (Chenopodiaceae). Novon 6, 398-403.

Mosyakin, S. L., Clements, S. E., 2002: New nomenclatural combinations in Dysphania R. Br. (Chenopodiaceae): taxa occurring in North America. Ukrainsky Botanichny Zhurnal 59, 380-385.

Mosyakin, S. L., Clements, S. E., 2008: Further transfers of glandular-pubescent species from Chenopodium subg. Ambrosia to Dysphania (Chenopodiaceae). Journal of the Botanical Research Institute of Texas 2, 425-431.

Mulligan, G., 1961: Chromosome numbers of Canadian weeds, 3. Canadian Journal of Botany 39, 1057-1066.

Murín, A., Ferakova, J. 1974: Chenopodium L. In: Majovsky et al. (eds), Index of chromosome numbers of Slovakian flora, 3. Acta Facultatis Rerum Naturalium Universitatis Comenianae 22, 1-20.

QueIros, M. 1975: Contribution to the cytotaxonomic knowledge of the Spermatophyta in Portugal, 10. Chenopodiaceae (in Portuguese). Boletim da Sociedade Broteriana, Série $2,121-142$.

RAHIMENIJAD, M. R. 2004: Chenopodium pumilio (Chenopodiaceae) new to the flora of Iran. Willdenovia 34, 183-186.

Raghavan, R., Arora, C. 1958: Chromosome numbers in Indian medicinal plants, 2. Proceedings of the Indian Academy of Sciences, Section B 47, 352-358.

Reynolds, J., F., CRAwFord, D. J., 1980: A quantitative study of variation in the Chenopodium atrovirens-desiccatum-pratericola complex. American Journal of Botany 67, 1380-1390.

Sharma, A. K., DeY., 1967: A comprehensive cytotaxonomic study on the family Chenopodiaceae. Journal of Cytology and Genetics 2, 114-127.

Schwarzova, T. 1978a: Reports. In: Löve, Å. (ed.), IOPB chromosome number reports 61 , Taxon 27, 375-392.

Schwarzova, T. 1978b: Chenopodium L. In: Majovsky at al. (eds), Index of chromosome numbers of Slovakian flora (part 6), Acta Facultatis Rerum Naturalium Universitatis Comenianae 26, 1-42.

Schwarzova, T. 1980: Reports. In: Löve, Å. (ed.), IOPB chromosome number report 69. Taxon 29, 728. 
Schwarzova, T. 1986: Chromosome numbers of some species of the genus Chenopodium L. from localities in Czechoslovakia. Acta Facultatis Rerum Naturalium Universitatis Comenianae 33, 37-40.

SchwarzovA, T. 1993: Beitrag zur Karyotaxonomie und Evolution mancher Arten der Gattung Chenopodium L. (Sektion Botryoides und Ambrina). Biologia (Bratislava) 48, 421-427.

Silvestre, S. 1984: Chromosome numbers 353-362, In: Choromosome numbers of Spanish flora (in Spanish), 300-364. Lagascalia 12, 298-303.

Uotila, P. 1973: Chromosome counts on Chenopodium L. from SE Europa and SW Asia. Annales Botanici Fennici 10, 337-340.

Uotila, P. 2001: Chenopodium L. In: Jonsell, B. (ed.), Flora Nordica, 2, 4-31. Bergius Foundation, Stockholm.

Uotila, P., SuOminen, J. 1976: The Chenopodium species in Finland, their occurrence and means of immigration. Annales Botanici Fennici 13, 1-25.

Vladimirov, V., Petrova, A., 2010: Reports 190-203. In: Vladimirov, V., Dane, F., SteVANOvić, V., TAN, K. (eds.), New floristic records in the Balkans, 14. Phytologia Balcanica $16,441-443$.

VladimiRov, V., 2011: Reports 124-130. In: VladimiRov, V., Dane, F., TAN, K. (eds.), New floristic records in the Balkans, 17. Phytologia Balcanica 17, 379-380.

Woroschilov, W. 1942: Review of species of Chenopodium L. from section Ambrina (Spach) Hook (in Russian). Botanichesky Zhurnal 27, 33-47.

Zhu, G., Mosyakin, S. L., Clements, S. E. 2003: Chenopodiaceae: Dysphania R. Br. In: Wu, Z. Y., RAven, P. H., Hong, D. Y. (eds), Flora of China, 5. Ulmaceae through Basellaceae, 376-378. Science Press, Beijing and Missori Botanical Garden Press, St. Louis. 